\title{
EUS-guided fine-needle core liver biopsy with a modified one-pass, one-actuation wet suction technique comparing two types of EUS core needles
}

\section{(ㄷ) (i) $(-)$}

\author{
Authors \\ Institutions \\ 1 Borland Groover Clinic, Jacksonville, Florida, United \\ States \\ 2 New York Presbyterian Hospital Weil Cornell - Joan and \\ Sanford I. Weill Department of Medicine, New York, NY, \\ United States \\ 3 Midwestern University, Downers Grove, Illinois, United \\ States \\ 4 University of Utah School of Medicine Huntsman Cancer \\ Center - Gastroenterology and Hepatology, Salt Lake \\ City, Utah, United States \\ 5 University of California at Los Angeles - Medicine, Los \\ Angeles, California, United States \\ submitted 17.11 .2019 \\ accepted after revision 9.4.2020 \\ Bibliography \\ DOI https://doi.org/10.1055/a-1165-1767 | \\ Endoscopy International Open 2020; 08: E938-E943 \\ (c) Georg Thieme Verlag KG Stuttgart · New York \\ eISSN 2196-9736 \\ Corresponding author \\ Jose Nieto, DO, New York Presbyterian Hospital Weill \\ Cornell - Joan and Sanford I. Weill Department of Medicine, \\ 1320 York Avenue, HT-621, New York, NY 10021 \\ Fax: +1-212-746-4007 \\ drjnieto@gmail.com
}

Jose Nieto*, 1, Enad Dawod*,2, Ameya Deshmukh³, Eli Penn¹, Douglas Adler4, Sammy Saab ${ }^{5}$

\section{ABSTRACT}

Background and study aims We compared the diagnostic yield and specimen adequacy in EUS-guided parenchymal biopsies between two types of EUS $19 \mathrm{G}$ core needles. Patients and methods This is a retrospective study of 420 patients at two tertiary medical centers in Florida with unexplained abnormal liver associated tests were referred for EUS evaluation of biliary obstruction and pancreatic pathology. EUS-guided liver biopsy (EUS-LB) was performed at the same session after biliary obstruction was excluded. We compared intact specimen length (ISL), total specimen length (TSL), complete portal triads (CPT) and adverse events (AE). Welch's T and Tukey tests were used for ISL, TSL and CPT.

Results A total of 210 patients underwent EUS-LB using a Franseen needle, 210 patients using a fork-tip needle. Median patient age was 52 years (15.63) and 238 (56.7\%) were female. The fork-tip needle had a mean ISL of 2.7 (1.1 SD) $\mathrm{cm}$, TSL of $6 \mathrm{~cm}$ (2.1SD), and mean 19.5 CPT (8.5SD) Abdominal pain occurred in 35 patients (17\%) post-procedure and was managed with supportive care. Two patients required intravenous (IV) narcotic administration. Subcapsular hematomas occurred in $1(0.5 \%)$ patients. The Franseen needle had a mean ISL of $3.1 \mathrm{~cm}(1.3 \mathrm{SD})$, TSL of $6.5 \mathrm{~cm}$ (2.6SD), and mean of 24 CPT (8.8SD). Abdominal pain occurred in four patients ( $2 \%$ ) post-procedure, which resolved in all patients after IV narcotic administration. Subcapsular hematomas occurred in $1(0.5 \%)$ and bile leak in 1 (0.4\%) patients.

Conclusions Use of the Franseen needle resulted in better liver core samples than that obtained with a fork-tip needle.

\section{Introduction}

Fibrosis is the hallmark of liver damage. It is the single most important predictor of outcomes in patients with underling liver disease. Historically, severity of fibrosis has been defined by percutaneous liver biopsies. Because of their invasive nature, such biopsies have been replaced by laboratory and imaging noninvasive markers of fibrosis. Although these tools are helpful in identifying patients with advanced fibrosis, they are limited in their ability to estimate fibrosis severity in patients with intermediate amounts of liver damage. Nevertheless, liver biopsy remains an important tool in selection of individuals [1-3].

\footnotetext{
* These authors contributed equally.
} 
Endoscopic ultrasound-guided liver biopsy (EUS-LB) is an emerging procedure that offers an alternative to conventional percutaneous and transjugular liver biopsy approaches $[4,5]$. EUS-guided liver biopsy has been shown to be efficacious in diagnosing both malignant and parenchymal liver disease. The safety and efficacy of EUS-LB in liver sampling and confirming histopathological diagnosis has been evaluated in a number of studies [6-8]. EUS has also demonstrated great ability in further characterization and staging of a previously detected lesion, which can impact disease management [9-12].

When parenchymal liver disease is unexplained by imaging or laboratory testing, EUS offers the advantage of avoiding sampling error, which is usually an issue with the conventional PC method because both the right and left lobes of the liver can be sampled during EUS. EUS-LB has also proven to provide excellent histologic specimens with regards to barometers of adequacy of histological yield specimen size, specimen length and complete portal tracts (CPT). By allowing simultaneous, live endoscopic and endosonographic visualization, accidental injury to adjacent blood vessels and/or organs is more easily avoided [13-15].

Newer flexible core needles have made it possible to obtain core parenchymal tissue. We previously demonstrated that EUS-modified LB (EUS-MLB), as described below, is a safe and effective method of obtaining adequate liver tissue [7]. Samples obtained using EUS-MLB in that study met the minimum measurement requirements that have been set by liver society organizations for CPT and specimen length [13].

In this study, we compared two types of core needles: Franseen tip fork-tip. Given the differences in their designs, we hypothesized that liver parenchymal yield, too, would differ. Thus, we sought to compare the histological yield of the two needles and the adverse events (AEs) associated with each needle type.

\section{Patients and methods}

We retrospectively studied 420 patients with unexplained abnormal liver-associated tests who were referred to two tertiary medical centers (both medical centers being in Florida) for evaluation with EUS. All the patients were referred for evaluation of the pancreas and biliary tree in the setting of unexplained elevated liver enzymes. The first 210 consecutive patients underwent EUS-MLB using a fork-tip needle, 165 of whom were also included in the previously published study. The next 210 patients underwent EUS-MLB using the Franseen tip needle. The decision to perform EUS-guided MLB was made by the endoscopist at the time of the procedure. EUS-guided liver biopsy was performed during the same session once pancreatobiliary pathology was excluded.

The EUS-MLB procedure was performed by one of two advanced endoscopists (JN, EP), both with substantial experience in endoscopic ultrasound. The two endoscopists reviewed all the imaging and pertinent clinical data before performing the procedures. Institutional review board approval was obtained.

\section{Technique}

\section{EUS-modified liver biopsy sampling}

In all procedures, monitored anesthesia care or general anesthesia was administered at the discretion of the certified anesthesia provider and cardiorespiratory monitoring was done by an anesthesiologist or a certified registered nurse anesthetist. A complete EUS examination for the primary procedure indication was performed. A standard linear-array echoendoscope was utilized to perform echoendoscopy. In addition, a complete EUS evaluation of the visualized liver and upper abdominal region was done to rule out varices or tumor.

EUS-MLB was performed once biliary and pancreatic pathology had been excluded. It was done using a novel, commercially available 19-gauge needle (Sharkcore or Acquire). The needle was primed with saline solution. The left lobe of the liver was accessed by the transgastric route and the right lobe by the transduodenal route. Under direct EUS guidance, the liver was punctured such that $7 \mathrm{~cm}$ of the needle was within the liver parenchyma. Next maximal $(20 \mathrm{~mL})$ suction was applied via a syringe.

The needle was then slowly withdrawn $3 \mathrm{~cm}$ back or if saline is displaced into the syringe then suction is turned off. With EUS guidance, large vessels can be avoided using Doppler imaging.

Wet suction or a saline prime needle was used to indicate tissue acquisition into the bore of the needle by displacing the saline solution into the syringe; this would notify the endoscopist to turn off the suction.

Core biopsies were immediately placed into formalin. The endoscopist personally separated the blood clots from the specimen and embedded paraffin in one container. This was followed by tissue block staining with hematoxylin and eosin. Additional immunohistochemistry was performed as needed per pathology. Experienced gastrointestinal pathologists examined the samples and evaluated for the outcome parameters mentioned below ( $\triangleright$ Fig. $\mathbf{1}$ ).

Patients were monitored in the endoscopy suite for at least 30 minutes to 1 hour following the procedure. Those who developed pain after the procedure were observed for a longer period of time. All patients were contacted by phone 48 hours after the procedure. Furthermore, patients were seen in clinic 2 weeks and 4 weeks after the procedure to assess for any AEs. A

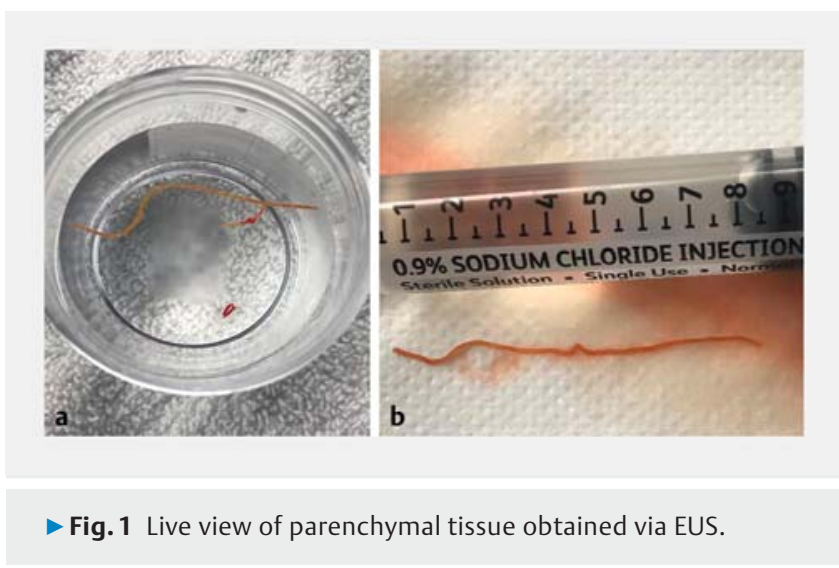


1-hour recovery time after the EUS-MLB is sufficient in almost all cases [7].

Technical success was defined as successful completion of all the steps of the EUS-modified liver biopsy sampling as outlined in the technique section above from the beginning of the EUS exam to completion of the tissue acquisition process. Clinical success was defined as successful tissue acquisition without AEs.

AEs were defined as any deviation from the anticipated intraprocedure and post-procedural course. AEs included but were not limited to bleeding, perforation, infection, bile leak, pneumothorax, and anesthesia-related events. Patients were followed for 2 weeks to 4 weeks to monitor for AEs.

Outcome parameters measured included total specimen length (TSL), intact specimen length, and complete portal tracts (CPTs), and rate of AEs. Furthermore, the ability of each needle to distinguish the different fibrosis scores was compared. Patient biopsies were divided according to their corresponding fibrosis scores: F0-F2 were compared alone and F3-F4 were compared separately.

\section{Statistical analysis}

Descriptive statistics were used to illustrate categorical and continuous variables. The ISL, TSL and CPTs were measured. Both Levene's and Bonett's tests were used to compare the variances between needle type and TSL, ISL, and CPT means. Equal Variance was only found for mean CPT. The findings of unequal variance required the use of Welch's T test and Games-Howell pairwise comparisons for TSL and ISL groups. Tukey and Fisher pairwise comparisons were used to compare needle type and CPT. One-way ANOVA was used to compare TSL, ISL and CPT groups between both needle types. $P<0.05$ was considered statistically significant. Post hoc power analyses for overall needle comparison, fibrosis score $0-2$ (ISL, TSL, CPT) and fibrosis score 3 to 4 (ISL, TSL, CPT) were completed.

\section{Results}

This was a retrospective study of 420 patients who underwent EUS-MLB at two medical centers between 2015 and 2018 for evaluation of pancreatic biliary pathology in the setting of unexplained elevated liver enzymes. The majority of patients were women (238/420). Mean age (SD) in both the fork-tip and Franseen groups was 52 (15.63). The patients were divided into two groups. The first group consisted of 210 patients who had undergone EUS-MLB using a 19-gauge fork-tip needle consecutively. The second group included 210 patients who underwent EUS-MLB using a 19 Franseen tip needle. The two groups were compared in terms of outcomes and histological yield.

The Franseen and fork-tip needle-types were used in 210 patients each, equating to 420 EUS-guided parenchymal biopsies in total. Each patient underwent two passes using the same needle type. Two hundred ninety-four patients (70\%) who received EUS-MLB had a history of viral hepatitis (96 patients) and fatty liver (198 patients). The other 126 patients (30\%) did not have any history of liver disease. Ninety-two patients (22\%) reported alcohol use. Fibrosis scores, mean TSL, ISL, and
CPT were compared as well as the percentages diagnosed with nonalcoholic fatty liver disease, viral hepatitis, and autoimmune conditions.

Needle type comparison for TSL, ISL, and CPT first occurred with inclusion of all patient types. Then patients were divided based on their corresponding fibrosis score for subsequent comparison of both needles for each one of the barometers of specimen adequacy.

\section{Total specimen length}

The mean (SD) TSL was statistically significant longer for forktip $(N=210)$ than for Franseen needles $(N=210)(5.99 \mathrm{~cm}$ [2.07] and $6.52 \mathrm{~cm}$ [2.56], respectively) $(P=0.02)$.

Mean (SD) TSL was $6.173 \mathrm{~cm}$ (2.03) and $6.232 \mathrm{~cm}$ (2.51) between the fork-tip $(\mathrm{N}=81)$ and Franseen $(\mathrm{N}=74)$ needles, respectively, for patients noted to have a histologic fibrosis score between 0 and $2(P=0.87)$ ( Table 1$)$. Mean TSL for forktip $(N=27)(6.97 \mathrm{~cm}[1.78])$ and Franseen $(N=52)(6.94 \mathrm{~cm}$ [2.80]) for patients with fibrosis scores of 3 or 4 was also similar $(P=-0.94)$.

Post hoc power analysis revealed a power score of $58 \%$ for overall comparison between fork-tip and Franseen needle types. This highlights that in future studies with larger sample sizes, the possibility of larger differences between groups may occur.

A power score of $3.6 \%$ was calculated for the fibrosis score 0 to 2 group. A power score of $3.0 \%$ was also found for the fibrosis score 3 to 4 group. The results of the power analysis indicate the need for larger sample sizes for comparison of fibrosis groups to improve detection of differences.

$\checkmark$ Table 1 Comparison of outcomes between two needles.

\begin{tabular}{|l|c|c|c|}
\hline & $\begin{array}{l}\text { Fork-tip } \\
\text { needle }\end{array}$ & $\begin{array}{l}\text { Franseen } \\
\text { needle }\end{array}$ & P value \\
\hline Overall & & & \\
\hline Mean TSL (SD) (cm) & $6.0(2.07)$ & $6.5(2.56)$ & 0.02 \\
\hline Mean ISL (SD) (cm) & $2.7(1.14)$ & $3.1(1.39)$ & 0.01 \\
\hline Mean CPT (SD) & $19.5(8.55)$ & $24.0(8.81)$ & $<0.01$ \\
\hline F0-2 & & & \\
\hline Mean TSL (SD) (cm) & $6.17(2.03)$ & $6.23(2.51)$ & 0.23 \\
\hline Mean ISL (SD) (cm) & $2.64(1.21)$ & $2.92(1.31)$ & 0.87 \\
\hline Mean CPT (SD) & $18.20(6.64)$ & $24.18(8.95)$ & $<0.01$ \\
\hline F3-4 & & & \\
\hline Mean TSL (SD) (cm) & $6.97(1.78)$ & $6.94(2.79)$ & 0.94 \\
\hline Mean ISL (SD) (cm) & $3.00(0.92)$ & $3.33(1.44)$ & 0.23 \\
\hline Mean CPT (SD) & $19.81(7.09)$ & $25.27(8.96)$ & $<0.01$ \\
\hline SD, standard deviation; TSL, total specimen length; ISL intact specimen \\
\hline length; CPT, complete portal tracts & & \\
\hline & & & \\
\hline
\end{tabular}




\section{Intact specimen length}

Comparison of ISL between fork-tip $(\mathrm{N}=210)$ and Franseen $(\mathrm{N}=$ $210)$ needles in all patients showed a statistically significant difference $(P=0.01)$ between means (fork-tip $2.67 \mathrm{~cm}$, Franseen $3.10 \mathrm{~cm})$.

Patients noted to have a histological fibrosis score between 0 and 2, had a mean ISL of $2.64 \mathrm{~cm}$ and $2.92 \mathrm{~cm}$ using the forktip $(\mathrm{N}=81)$ and Franseen $(\mathrm{N}=76)$ needles, respectively $(P=$ $0.17)$. Patients falling into fibrosis scores of 3 to 4 did not have a statistically significant difference $(P=0.23)$ in the mean ISL between fork-tip $(\mathrm{N}=27)(3.00 \mathrm{~cm})$ and Franseen $(\mathrm{N}=52)$ $(3.33 \mathrm{~cm})(\triangleright$ Table 1$)$.

Post hoc power analysis revealed a score of $92.6 \%$. This indicates a sufficiently large sample size was used in the comparison of means between groups.

Power analysis calculated a power of $28.5 \%$ for fibrosis scores between 0 and 2 . A power score of $22.9 \%$ was also found for the fibrosis scores between 3 and 4 . The results of the power analysis indicate the need for larger sample sizes for comparison of fibrosis groups to improve detection of differences.

\section{Complete portal tracts}

More CPTs could be obtained with the Franseen needle than with the Fork-tip needle, which aids the pathologist in making a more accurate diagnosis during biopsy analysis.

There was a statistically significantly greater number of CPTs noted with Franseen $(\mathrm{N}=210)$ as compared to fork-tip ( $N=210)$ needles (mean 24.05 vs 19.55 , respectively) $(P<0.01)$.

The mean CPT was 18.198 and 24.18 between the fork-tip $(\mathrm{N}=81)$ and Franseen $(\mathrm{N}=76)$ needles, respectively, in patients with fibrosis scores between 0 and 2 . This difference was statistically significant $(P<0.01)$. For patients with fibrosis scores of 3 to 4 did, a statistically significant difference $(P<0.01)$ was seen in the mean CPT between fork-tip ( $N=27)(19.81)$ and Franseen $(\mathrm{N}=52)(25.27)$ ( $\triangleright$ Table 1$)$.

Post hoc power analysis revealed a score of $100 \%$ for the overall comparison between needle types, indicating an adequate sample size was used in the comparison of means between groups. A power score of $99.7 \%$ was calculated for patients with fibrosis scores 0 to 2 . The power score was $84.1 \%$ for those with fibrosis scores 0 to 3 , which also demonstrates a satisfactory sample size.

\section{Adverse events}

The number of AEs were comparable between both needle types. EUS-liver biopsy utilizing the fork-tip needle was associated with abdominal pain in 35 patients (17\%) immediately post-procedure. Two patients required intravenous (IV) narcotic administration (hydromorphone $0.25 \mathrm{mg}$ IVP every 2 hours as needed for pain) for a total of . $5 \mathrm{mg}$ and $1 \mathrm{mg}$ in each patient, respectively. In addition, subcapsular hematomas developed in one patients ( $0.5 \%$ ). The hematoma measured $2.5 \mathrm{~cm}$ by $3.5 \mathrm{~cm}$ on abdominal computed tomography. The patient was admitted overnight for pain control and received 1 unit of packed red blood cell transfusion. The hematoma resolved on followup abdominal imaging 1 month later.
- Table 2 Baseline characteristics and demographic data according to needle type.

\begin{tabular}{|l|c|c|}
\hline & Franseen & Fork-tip \\
\hline Male \% & 44 & 43 \\
\hline Age (SD) & $52(7.9)$ & $52(16.6)$ \\
\hline Mean TSL (cm)(SD) & $6.5(2.6)$ & $6.0(2.1)$ \\
\hline Mean ISL (cm)(SD) & $3.1(1.3)$ & $2.7(1.1)$ \\
\hline Mean CPT (SD) & $24(8.8)$ & $19.5(8.5)$ \\
\hline Major AE & 0 & 1 \\
\hline \% Diagnosed NASH & 61 & 50 \\
\hline \% Diagnosed viral hepatitis & 10 & 19 \\
\hline \% Diagnosed autoimmune & 0.5 & 2 \\
\hline \% Alcohol use & 23 & 21 \\
\hline Mean BMI (SD) & $32.2(15.2)$ & $29.6(7.4)$ \\
\hline $\begin{array}{l}\text { Mean platelet count } \\
\text { length; CPT, complete portal tracts; NASH, nonalcoholic steatohepatitis; }\end{array}$ \\
\hline \begin{tabular}{l} 
Mean INR \\
\hline SD, body mass index; INR, international normalized ratio
\end{tabular} \\
\hline
\end{tabular}

Four patients $(2.0 \%)$ developed abdominal pain following the biopsy when using the Franseen needle, which resolved in all of them after IV narcotic administration. Bile leak occurred in one patient $(0.4 \%)$ patient $(\triangleright$ Table 2$)$.

\section{Discussion}

The technique of EUS-LB has evolved. The first core needle used to obtain core biopsy specimen was the Quick-Core (Cook Medical Inc, Bloomington, Indiana, United States) by means of a spring-loaded trucut mechanism [16]. We recently published an article on use of 19-gauge needles in obtaining parenchymal cores with a modified one-pass, one actuation wet suction technique. One hundred sixty five of the patients included in this study were also included in the previously published study [7]. Since then multiple studies have shown the utility of $19 \mathrm{G}$ and $22 \mathrm{G}$ core needles in EUS-MLB. The Acquire needle's design consisting of the three-point heel allows maximum tissue capture and minimal fragmentation. On the other hand, the Sharkcore needle design consists of six distal cutting edges, which facilitates obtaining cohesive units of tissue with preserved cell architecture [4].

Using a modified one-pass, one-actuation wet suction technique, both needles met $100 \%$ American Association for the Study of Liver Dieases specimen adequacy (>11 CPT or TSL $>2.5 \mathrm{~cm}$ ). For patients with fibrosis scores of 1 to 2 , the Franseen needles were capable of retrieving more CPTs. The difference persisted in patients with fibrosis scores of 3 to 4 as the Franseen needle was also able to obtain more CPTs. For all fibrosis scores, there was no difference in the needles in terms of TSL 
and ISL. Overall comparison including all patient types showed that the Franseen needle was able to obtain a larger TSL and ISL and more CPTs (> Fig. 2, > Fig. 3 ).

In a recent meta-analysis published by Mohan et al., 437 patients who had undergone EUS-LB were studied [4]. The yield was $93.9 \%$ and the $A E$ rate was $2.3 \%$, proving the safety and efficacy of EUS-LB. A subgroup analysis compared the outcomes based on the type of biopsy needle. The AE rate was found to be lower with the 19-gauge FNA needle when compared with core biopsy needles and the diagnostic yield was also higher. On further subgroup analysis, the rate of diagnostic yield was not statistically significantly different between the 19-gauge FNA needle and core needles. The FNA needle had a significantly lower rate of insufficient specimens than core biopsy needles (QuickCore and ProCore) [4].

In terms of AEs, the rates were comparable between both groups in our study. In our experience, bleeding as a complication of EUS-MLB is a rare occurrence. Only one patient developed a subcapsular hematoma, which eventually resolved. With EUS-MLB, a 1-hour period after the procedure is usually sufficient for pain to subside [7]. Unlike with PC liver biopsy, the fact that patients undergoing EUS are usually sedated eliminates problems with patient cooperation, which would otherwise raise the risk of bleeding. The 1 hour proved to be sufficient to achieve pain relief according to the meta-analysis by Mohan et al [17]. In the 437 patients who underwent EUS-MLB included in the meta-analysis, the pooled rate of AEs with EUSguided LB was $2.3 \%$. Sub-analysis showed that the rate of AEs, including pain, with the 19-gauge FNA needle was not significantly different than the rate of AEs with other biopsy needles.

To the best of our knowledge, this cohort of 420 patients is the largest study evaluating endoscopic ultrasound for obtaining liver samples using core needles. Previous studies have compared 19G fine-needle aspiration (FNA) with 19G fine-needle biopsy (FNB), or 19G FNA needle with a 22G core needle (SharkCore) $[18,19]$. Comparing the 19G FNA versus 19G FNB, the core needle demonstrated superiority in regard to all measures of liver biopsy [19]. Comparing the 19G FNA to 22G core, the $22 \mathrm{G}$ had higher rates of tissue fragmentation which lead to lower tissue adequacy rates compared to 19G FNA [18]. Dewitt et al compared two $19 \mathrm{G}$ core needles in 85 patients and demonstrated superior ability of FNB compared to Tru-Cut biopsy needle in terms of prevalence of diagnostic histology, TSL, and CPT [20]. In a recent study by Hasan MK et al, efficacy and safety of 22-G EUS-FNB for performing EUS-MLB was evaluated. Forty patients were included in the study and in $100 \%$ of them, a 22-G needle was able to provide sufficient unfragmented core tissue with $100 \%$ histological diagnosis of parenchymal liver disease [21]. Our current study with 420 patients is the first head-to-head study of its size to compare different two kinds of core needles for obtaining tissue in evaluation of parenchymal liver disease.

Our study has several important limitations. First, the results are limited by its retrospective nature. However, the biopsies were contemporaneously related and done at two different institutions. Another limitation is that the results (technical success, CPT, tissue length) may not generalizable to all endos-

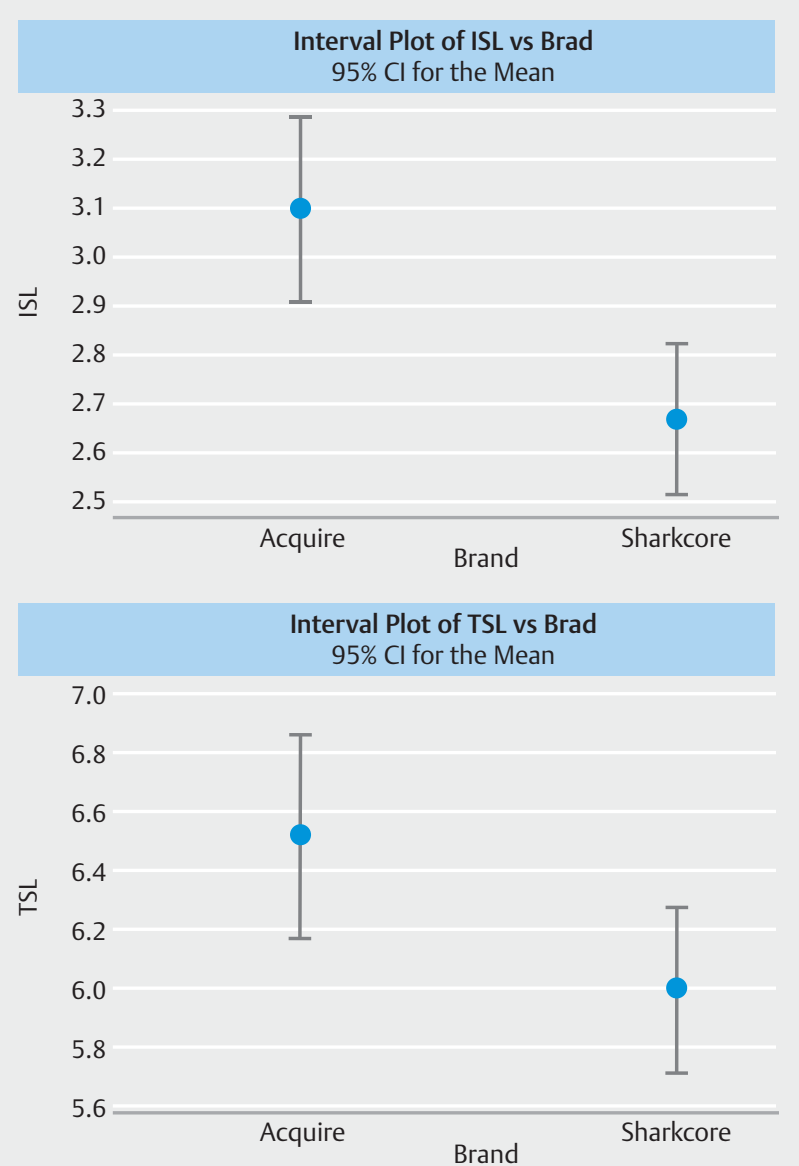

Individual standard deviations are used to calculate the intervals.

- Fig. 2 Mean intact specimen length (ISL) and total specimen length (TSL) comparison with inclusion of all patient types.

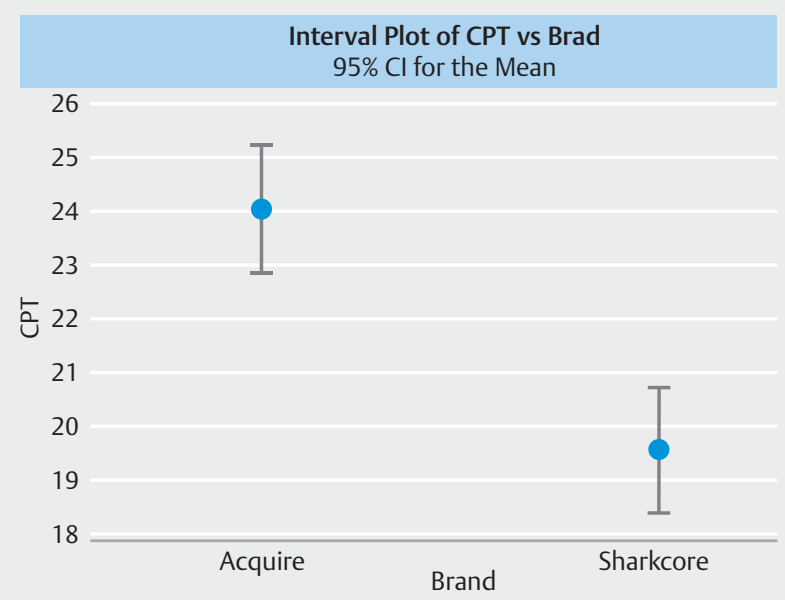

The pooled standard deviation is used to calculate the intervals.

- Fig. 3 Comparison with inclusion of all patient types between the two needles in terms of complete portal tracts (CPT). 
copists. EUS-MLB is a technically demanding procedure and preference should be given to placing it in the hands of expert endoscopists at tertiary medical centers where there is high volume and more familiarity with EUS-MLB so as to be able to replicate findings reported in this study and recent literature. Nevertheless, the results highlight the overall safety and efficacy using one cohort evaluated.

\section{Conclusion}

In conclusion, our results indicate a difference between the needles in terms of TSL, ISL and CPT. However, histological yield was not affected and both needles were able to provide sufficient tissue for histological analysis in all patients regardless of fibrosis score. However, since the Franseen tip obtains more specimen, EUS procedures performed with it may be reduced to only one pass, therefore, possibly reducing complications and procedure times. There is a need for randomized clinical trials to establish the superiority of one needle over the other. Moreover, the yield from EUS-MLB will also likely increase as the technique for the procedure is further perfected with continued research and technological innovation. Wider application of EUS-MLB outside the scope of tertiary medical centers with advanced endoscopy services will also contribute to perfecting the technique and its histological yield.

\section{Competing interests}

Dr. Nieto is a consultant for Boston Scientific and Olympus. Dr. Adler is a consultant for Boston Scientific and Olympus.

\section{References}

[1] Sheen V, Nguyen H, Jimenez M et al. Routine laboratory blood tests may diagnose significant fibrosis in liver transplant recipients with chronic hepatitis C: a 10 year experience. J Clin Transl Hepatol 2016; 4: 20-25

[2] Rockey DC, Caldwell SH, Goodman ZD et al. American Association for the Study of Liver Diseases. Liver biopsy. Hepatology 2009; 49: 10171044

[3] Cross T], Calvaruso V, Maimone S et al. Prospective comparison of Fibroscan, King's score and liver biopsy for the assessment of cirrhosis in chronic hepatitis C infection. J Viral Hepat 2010; 17: 546-554

[4] Mohan BP, Shakhatreh M, Garg R et al. Efficacy and safety of EUSguided liver biopsy: a systematic review and meta-analysis. Gastrointest Endosc 2019; 89: 238-246.e3
[5] Shiha G, Ibrahim A, Helmy A et al. Asian-Pacific Association for the Study of the Liver (APASL) consensus guidelines on invasive and noninvasive assessment of hepatic fibrosis: a 2016 update. Hepatol Int 2017; 11: 1-30

[6] Saab S, Phan J, Jimenez MA et al. endoscopic ultrasound liver biopsies accurately predict the presence of fibrosis in patients with fatty liver. Clin Gastroenterol Hepatol 2017; 15: 1477-1478

[7] Nieto J, Khaleel H, Challita Y et al. EUS-guided fine-needle core liver biopsy sampling using a novel 19-gauge needle with modified 1-pass, 1 actuation wet suction technique. Gastrointest Endosc 2018; 87: 469-475

[8] Diehl DL, Johal AS, Khara HS et al. Endoscopic ultrasound-guided liver biopsy: a multicenter experience. Endosc Int Open 2015; 3: E210E215

[9] Oh D, Seo DW, Hong SM et al. Endoscopic ultrasound-guided fineneedle aspiration can target right liver mass. Endosc Ultrasound 2017; 6: 109-115

[10] Saraireh HA, Bilal M, Singh S. Role of endoscopic ultrasound in liver disease: Where do we stand in 2017? World J Hepatol 2017; 29: 1013-1021

[11] Singh P, Mukhopadhyay P, Bhatt B et al. Endoscopic ultrasound versus CT scan for detection of the metastases to the liver: results of a prospective comparative study. J Clin Gastroenterol 2009; 43: 367-373

[12] Parekh PJ, Majithia R, Diehl DL et al. Endoscopic ultrasound-guided liver biopsy. Endosc Ultrasound 2015; 4: 85-91

[13] Bravo AA, Sheth SGS. Liver biopsy. N Engl J Med 2001; 344: 495-500

[14] Magalhães J, Monteiro S, Xavier S et al. Endoscopic ultrasonography emerging applications in hepatology. World J Gastrointest Endosc 2017; 9: 378-388

[15] Gleeson FC, Clayton AC, Zhang L et al. Adequacy of endoscopic ultrasound core needle biopsy specimen of nonmalignant hepatic parenchymal disease. Clin Gastroenterol Hepatol 2008; 6: 1437-1440

[16] Schulman AR, Thompson CC, Odze R et al. Optimizing EUS-guided liver biopsy sampling: comprehensive assessment of needle types and tissue acquisition techniques. Gastrointst Endosc 2017; 85: 419-426

[17] Mok SRS, Diehl DL. The role of EUS in liver biopsy. Curr Gastroenterol Rep 2019; 21: 6

[18] Mok SR, Dielh DL, Johal AS et al. Mo1245 19 versus 22 gauge fine needle biopsy for endoscopic ultrasound guided liver biopsy (EUS-LB): a prospective randomized trial. Gastrointest Endosc 2017; 85: AB473-474

[19] Ching Companioni RA, Johal AS, Confer B et al. Mo1349 a prospective randomized trial of 19-gauge $(G)$ aspiration needle versus $19 \mathrm{G}$ core biopsy needle for endoscopic ultrasound- guided liver biopsy. Gastrointest Endosc 2018; 87: AB457-AB458

[20] DeWitt ], Cho CM, Lin J et al. Comparison of EUS-guided tissue acquisition using two different 19-gauge core biopsy needles: a multicenter, prospective, randomized, and blinded study. Endosc Int Open 2015; 3: E471-E478

[21] Hasan MK, Kadkhodayan K, Idrisov E et al. Endoscopic ultrasoundguided liver biopsy using a 22-G fine needle biopsy: a prospective study. Endoscopy 2019; 51: 818-824 